\title{
Electromagnetic field of a bunch intersecting a vacuum gap in a dielectric loaded waveguide
}

\author{
Tatiana Yu. Alekhina* and Andrey V. Tyukhtin ${ }^{\dagger}$ \\ Physical Faculty of St. Petersburg State University, St. Petersburg 198504, Russia
}

(Received 8 May 2014; published 31 July 2014)

\begin{abstract}
The electromagnetic field of a bunch moving through a vacuum gap located in a dielectric loaded waveguide is under investigation. This paper focuses on the case when Cherenkov radiation is generated in the dielectric parts of the waveguide. Analysis of the field components of the waveguide mode is performed both analytically and numerically, and the electromagnetic field structure for different time moments and different gap lengths is demonstrated. In particular, it is shown that for dielectrics with permittivity $\varepsilon<2$, the field of the ultrarelativistic bunch can be restored in the area after the vacuum gap for some optimal parameters of the problem. However, for dielectrics with $\varepsilon>2$, restoration of the wakefield can only be realized for small lengths of the gap.
\end{abstract}

DOI: 10.1103/PhysRevSTAB.17.071302

PACS numbers: 41.75.Lx, 41.60.Dk

\section{INTRODUCTION}

Investigation of the electromagnetic fields of charged particles in waveguides loaded with dielectrics is of interest in the development of new methods of generating electromagnetic radiation and accelerating charged particles (the so-called "wakefield acceleration") [1-3]. One of the difficulties in this area is that, whereas real dielectric tubes have relatively small lengths (not greater than several tens of centimeters), ideal scientific wakefield accelerators should consist of many dielectric sections. Because each gap between the sections is a source of distortions of the wakefield, the estimation of these effects is important.

This work is devoted to a three-layer waveguide structure consisting of a dielectric, a vacuum, and another dielectric (both dielectric layers are assumed to be semi-infinite longitudinally). It should be noted that, although the case of a dielectric plate situated in a waveguide (a vacuumdielectric-vacuum structure) has been analyzed before [4-6], it differs dramatically from the case considered here both mathematically and physically.

Furthermore, note that radiation of charged particles moving in a waveguide and crossing a single boundary between two homogenous media has also been studied in detail $[7,8]$. In particular, the effect of "Cherenkovtransition radiation" (CTR) has been investigated in the case of a dielectric-vacuum boundary [8]. It has been shown that the CTR can be dominant in the vacuum area under certain conditions. This holds also true for the

\footnotetext{
*tanya@niirf.spbu.ru

†yukhtin@bk.ru
}

Published by the American Physical Society under the terms of the Creative Commons Attribution 3.0 License. Further distribution of this work must maintain attribution to the author $(s)$ and the published article's title, journal citation, and DOI. dielectric plate [6]; however, the length of the plate should be quite large in order that the Cherenkov radiation (CR) is formed inside the plate. Thus, it is interesting to consider the situation when the CTR penetrates the next vacuumdielectric boundary. As we will show here, owing to the CTR effect, the radiation field in the dielectric area after the vacuum cavity can be similar to the wakefield in an infinite regular waveguide loaded with a dielectric even for a large length of the vacuum gap. However, when the CTR in the vacuum gap is negligible, this effect only takes place for small lengths of the gap.

Here, we will calculate the electromagnetic field in both situations. Special attention will be paid to the field in the dielectric area after the vacuum cavity. In particular, we will reveal conditions for the "restoration" of the wakefield in this area.

\section{GENERAL SOLUTION}

Let us consider the following problem: a bunch of charged particles moves uniformly with a velocity $\vec{V}=$ $c \beta \vec{e}_{z}$ along the axis of a circular waveguide and intersects a vacuum cavity situated in a dielectric filling (Fig. 1). The waveguide radius is $a$, the dielectric permittivity is $\varepsilon$, and the length of the cavity is $d$. The bunch is characterized by a distribution along the $z$ axis and a negligible thickness, i.e., the charge density is $\rho=q \delta(x) \delta(y) \eta(\zeta)$, where

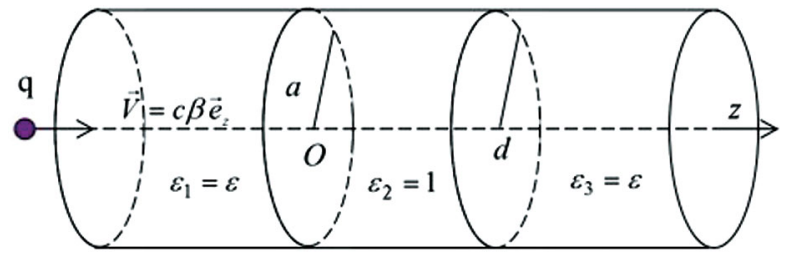

FIG. 1. The geometry of the problem. 


$$
\int_{-\infty}^{+\infty} \eta(\zeta) d \zeta=1, \quad \zeta=z-c \beta t
$$

For example, $\eta(\zeta)=\exp \left[-\zeta^{2} /\left(2 \sigma^{2}\right)\right] /(\sqrt{2 \pi} \sigma)$ for the bunch with the Gaussian distribution, where $\sigma$ is the half length of the bunch. The middle of the bunch intersects the first boundary $z=0$ at the moment $t=0$. We label the dielectric areas before and after the cavity as areas 1 and 3 , respectively (here the permittivity is $\varepsilon_{1}=\varepsilon_{3}=\varepsilon$ ). In addition, area 2 (the vacuum gap) is characterized by the permittivity $\varepsilon_{2}=1$. The media are nonmagnetic and have no dispersion.

The analytical solution to this problem is traditionally found via an expansion into a series of eigenfunctions of the transversal operator [4]. The electromagnetic field components in each medium have two summands:

$$
\vec{E}_{j}=\vec{E}_{j}^{q}+\vec{E}_{j}^{b},
$$

where $j=1,2,3$ is the number of the area. The first summand $\left(\vec{E}_{j}^{q}\right)$, which was called the "forced" field by
Ginzburg [9], is the field in an infinite regular waveguide (this field contains $\mathrm{CR}$ if the bunch velocity exceeds the Cherenkov threshold). The second summand $\left(\vec{E}_{j}^{b}\right)$ is the "free" field connected with the influence of the boundary, and it includes transition radiation (TR). The forced field has been thoroughly studied in the literature [10]. Hence, we pay the main attention to analyzing the free field components. The longitudinal components of the free field in each of the media [4] can be written in the following form:

$$
\begin{gathered}
E_{z j}^{b}=\frac{2 q c^{2}}{\pi a^{4} \varepsilon_{j}} \sum_{n=1}^{\infty} \frac{\chi_{n}^{2} J_{0}\left(\chi_{n} r / a\right)}{J_{1}^{2}\left(\chi_{n}\right)} I_{z j}^{b}, \\
I_{z j}^{b}=\frac{-i \beta(\varepsilon-1)}{\left(1-\beta^{2}\right)\left(1-\beta^{2} \varepsilon\right)} \sum_{l=1}^{4} \int_{-\infty}^{+\infty} \tilde{\eta}(\omega) f_{j l}(\omega) e^{F_{j l}(\omega)} d \omega,
\end{gathered}
$$

where $j=1,2,3, F_{j l}=-i \omega t+i A_{j l}$,

$$
\begin{aligned}
& \hat{f}=\frac{1}{q_{n} \Delta_{n}}\left(\begin{array}{cccc}
g_{n}^{+} r_{n}^{+}, & g_{n}^{-} r_{n}^{-}, & -2 c \varepsilon k_{2} p_{n}^{+}, & 0 \\
-g_{n}^{-} p_{n}^{+}, & g_{n}^{+} p_{n}^{-}, & -g_{n}^{+} p_{n}^{+}, & g_{n}^{-} p_{n}^{-} \\
-g_{n}^{+} r_{n}^{-}, & -g_{n}^{-} r_{n}^{+}, & 2 c \varepsilon k_{2} p_{n}^{-}, & 0
\end{array}\right), \\
& \hat{A}=\left(\begin{array}{cccc}
-k_{1} z-k_{2} d, & -k_{1} z+k_{2} d, & -k_{1} z+\frac{\omega d}{\beta c}, & 0 \\
k_{2} z+\frac{\omega d}{\beta c}, & k_{2} \tilde{z}, & -k_{2} z+\frac{\omega d}{\beta c}, & k_{2} \tilde{z} \\
k_{1} \tilde{z}-k_{2} d+\frac{\omega d}{\beta c}, & k_{1} \tilde{z}+k_{2} d+\frac{\omega d}{\beta c}, & k_{1} \tilde{z}, & 0
\end{array}\right) \text {, } \\
& g_{n}^{ \pm}=c\left(\varepsilon k_{2} \pm k_{1}\right) \text {, } \\
& \Delta_{n}=\left(g_{n}^{+}\right)^{2} e^{-i k_{2} d}-\left(g_{n}^{-}\right)^{2} e^{i k_{2} d} \text {, }
\end{aligned}
$$$$
q_{n}=\left[\omega^{2}+\beta^{2} \omega_{n}^{2}\left(1-\beta^{2}\right)^{-1}\right]\left[\omega^{2}+\beta^{2} \omega_{n}^{2}\left(1-\beta^{2} \varepsilon\right)^{-1}\right],
$$$$
\alpha_{n}=\omega^{2}\left(1-\beta^{2}-\beta^{2} \varepsilon\right)+\beta^{2} \omega_{n}^{2},
$$$$
r_{n}^{ \pm}=\alpha_{n} \pm \omega \beta^{3} \varepsilon c k_{2}, \quad p_{n}^{ \pm}=\alpha_{n} \pm \omega \beta^{3} c k_{1} .
$$$$
k_{1}=\sqrt{\omega^{2} \varepsilon-\omega_{n}^{2}} / c, \quad k_{2}=\sqrt{\omega^{2}-\omega_{n}^{2}} / c,
$$

$\tilde{z}=z-d, \omega_{n}=\chi_{n} c / a, \chi_{n}$ is the $n$-th zero of the Bessel function $\left[J_{0}\left(\chi_{n}\right)=0\right], \operatorname{Im} k_{1,2}>0$, and $\tilde{\eta}(\omega)$ is the Fourier transform of the longitudinal distribution normalized to $\exp [i \omega z /(\beta c)] /(2 \pi \beta c)\left[\tilde{\eta}(\omega)=\exp \left(-\frac{\omega^{2} \sigma^{2}}{2 \beta^{2} c^{2}}\right)\right.$ for the bunch with the Gaussian distribution].

We investigate the exact solution (2)-(6) both analytically and numerically. Analytical research is carried out with the methods of complex variable function theory. The computations are based on an algorithm that uses separation between the integration path and the singularities of the integrands. Then, we focus on the case when the condition for CR in the dielectric is satisfied, that is, when $\beta^{2} \varepsilon>1$.

\section{ANALYTICAL RESEARCH}

In the analytical method [11,12], the singularities of the integrands (3) are studied in a complex plane $(\omega)$. There are the following singularities: (i) two branch points $\pm \tilde{\omega}_{n}^{(1)}=$ $\pm \omega_{n} / \sqrt{\varepsilon}-i \delta_{1}$ (which are illustrated in Fig. 2 as circles) with a corresponding branch cut that is defined by the equation $\operatorname{Re} \sqrt{\omega^{2} \varepsilon-\omega_{n}^{2}}=0$. It should be noted that the points $\pm \omega_{n}$ in (6) are not the branch ones for the integrands considered as a whole; (ii) two poles $\pm \omega_{0 n}^{(2)}=$ $\pm i \beta \omega_{n}\left(1-\beta^{2}\right)^{-1 / 2}$, which are always situated on an imaginary axis (shown in Fig. 2 with crosses); (iii) two poles situated on the imaginary axis $\pm \omega_{0 n}^{(1)}= \pm i \beta \omega_{n}\left(1-\varepsilon \beta^{2}\right)^{-1 / 2}$ if $\beta<\varepsilon^{-1 / 2}$ or on the real axis $\pm \omega_{0 n}^{(1)}= \pm \beta \omega_{n}\left(\varepsilon \beta^{2}-1\right)^{-1 / 2}-$ $i \delta_{2}$ if $\beta>\varepsilon^{-1 / 2}$.

Here, $\delta_{1}, \delta_{2}$ are positive infinitesimal quantities that indicate that all of the singularities are located below the real axis if small losses in the media are taken into account. The poles $\pm \omega_{0 n}^{(1)}$ and $\pm \omega_{0 n}^{(2)}$ are the zeros of the function 


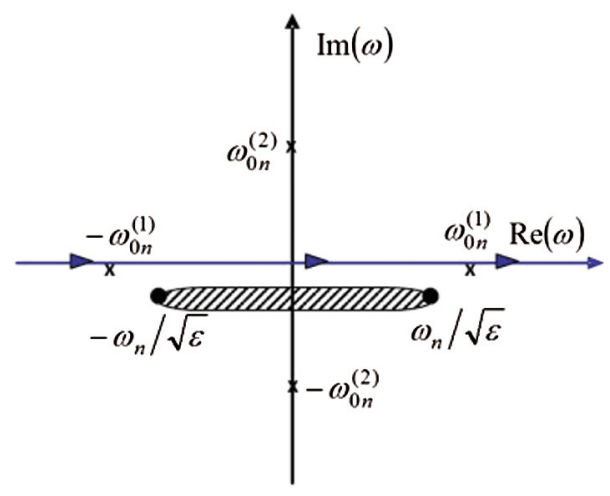

FIG. 2. The disposition of the singularities of the integrands, the branch cut and integration path in a complex plane $(\omega)$ for the free field components when $\beta>\varepsilon^{-1 / 2}$.

$q_{n}$ (5) in the denominators of the integrands. As opposed to the case of a dielectric plate situated in a vacuum $[4,6]$, here the function $\Delta_{n}$ (4) has no zeros, which might otherwise give contributions to the free field.
If the $\mathrm{CR}$ is generated in the dielectric, the contributions from the poles $\pm \omega_{0 n}^{(1)}$ to the free field give the CTR [7,8], which is the reflected and transmitted waves of the CR. These contributions can be evaluated by means of the residue theorem. It can be shown that the CTR can only exist in the vacuum gap under the conditions $\beta_{C}<\beta<\beta_{\mathrm{CT}}$, where the lower threshold $\beta_{C}=\varepsilon^{-1 / 2}$ is connected with the condition of the Cherenkov radiation generation and the upper threshold $\beta_{\mathrm{CT}}=(\varepsilon-1)^{-1 / 2}$ is explained by total internal reflection of the Cherenkov waves from the boundaries.

We obtain the expression for the CTR in all of the media in the following form:

$$
E_{z j}^{\mathrm{CTR}}=-\frac{4 q}{a^{2} \varepsilon} \sum_{n=1}^{\infty} \frac{J_{0}\left(\frac{\chi_{n} r}{a}\right)}{J_{1}^{2}\left(\chi_{n}\right)} \tilde{\eta}\left(\omega_{0 n}^{(1)}\right) I_{j}^{\mathrm{CTR}},
$$

where $j=1,2,3$,

$$
\begin{aligned}
I_{1}^{\mathrm{CTR}} & =\operatorname{Re}\left\{2 i \Lambda_{n}^{-1}\left(\varepsilon^{2} S^{2}-1\right) \exp \left[-i \kappa_{n}(z+\beta c t)\right] \sin \left(\kappa_{n} d S\right)\right\} \theta\left(Z_{1}-|z|\right), \\
I_{2}^{\mathrm{CTR}} & =2 \varepsilon \operatorname{Re}\left\{\Lambda_{n}^{-1} e^{-i \kappa_{n} \beta c t}\left[(\varepsilon S-1) e^{-i \kappa_{n} \tilde{z} S}+(\varepsilon S+1) e^{i \kappa_{n} z S}\right]\right\} \theta\left(Z_{2}-z\right), \\
I_{3}^{\mathrm{CTR}} & =\left\{-\cos \left(\kappa_{n} \zeta\right)+\operatorname{Re}\left[4 \varepsilon S \Lambda_{n}^{-1} e^{i \kappa_{n}(\zeta-d)}\right]\right\} \theta\left(Z_{3}-z\right), \\
\Lambda_{n} & =(\varepsilon S+1)^{2} e^{-i \kappa_{n} d S}-(\varepsilon S-1)^{2} e^{i \kappa_{n} d S}, \\
S & =\sqrt{1-\beta^{2}(\varepsilon-1)}, \quad \kappa_{n}=\chi_{n} a^{-1}\left(\varepsilon \beta^{2}-1\right)^{-1 / 2},
\end{aligned}
$$

and $\theta(\xi)$ is the Heaviside function. The CTR propagates in the dielectric area [7] with a group velocity

$$
\mathrm{v}_{\mathrm{g} 1}=c(\beta \varepsilon)^{-1}
$$

and in the vacuum area [8] with a group velocity

$$
\mathrm{v}_{\mathrm{g} 2}=c \beta^{-1} \sqrt{1-\beta^{2}(\varepsilon-1)} .
$$

Thus, by taking into account the finite length of the gap one can demonstrate that the fronts of the CTR depend on time. These fronts can be presented in the following form:

$$
\begin{aligned}
& Z_{1}(t)= \begin{cases}0 & \text { for } t<2 d / \mathrm{v}_{\mathrm{g} 2}, \\
\left(t-2 d / \mathrm{v}_{\mathrm{g} 2}\right) \mathrm{v}_{\mathrm{g} 1} & \text { for } t>2 d / \mathrm{v}_{\mathrm{g} 2},\end{cases} \\
& Z_{2}(t)= \begin{cases}t \mathrm{v}_{\mathrm{g} 2} & \text { for } t<d / \mathrm{v}_{\mathrm{g} 2}, \\
d & \text { for } t>d / \mathrm{v}_{\mathrm{g} 2},\end{cases} \\
& Z_{3}(t)= \begin{cases}d & \text { for } t<d / \mathrm{v}_{\mathrm{g} 2}, \\
\left(t-d / \mathrm{v}_{\mathrm{g} 2}\right) \mathrm{v}_{\mathrm{g} 1} & \text { for } t>d / \mathrm{v}_{\mathrm{g} 2} .\end{cases}
\end{aligned}
$$

As can be seen, the CTR (7) has two summands in the domain after the vacuum gap (area 3). Because the first summand equals the wakefield in the dielectric

$$
E_{z 1,3}^{\mathrm{CR}}=\frac{-4 q}{a^{2} \varepsilon} \sum_{n=1}^{\infty} \frac{J_{0}\left(\frac{\chi_{n} r}{a}\right)}{J_{1}^{2}\left(\chi_{n}\right)} \tilde{\eta}\left(\omega_{0 n}^{(1)}\right) \cos \left(\kappa_{n} \zeta\right)
$$

taken with the opposite sign, it compensates for the wakefield in some area after the vacuum cavity as in the case of a singular vacuum-dielectric boundary [7]. However, the second summand can restore the wakefield in medium 3, as will be shown below.

The investigation concerning the CTR (7) reveals two different situations: the case $\beta<\beta_{\mathrm{CT}}=(\varepsilon-1)^{-1 / 2}$, when the CTR effect takes place in the vacuum cavity, and the case $\beta>\beta_{\mathrm{CT}}$, when the CTR is absent in the vacuum cavity. For ultrarelativistic bunches, when $\beta \rightarrow 1$ [or the Lorentz factor $\gamma=\left(1-\beta^{2}\right)^{-1 / 2} \gg 1$ ], these situations correspond to the dielectric permittivities $\varepsilon<2$ and $\varepsilon>2$ accordingly.

If $\beta<\beta_{\mathrm{CT}}$, then $S$ is real in (8). One can obtain the following expressions:

$$
\begin{aligned}
I_{1}^{\mathrm{CTR}}= & C_{n 1} \cos \left[\Phi-\kappa_{n}(z+\beta c t)\right], \\
I_{2}^{\mathrm{CTR}}= & C_{n 21} \cos \left(\Phi+\kappa_{n} \tilde{z} S-\kappa_{n} \beta c t\right) \\
& +C_{n 22} \cos \left(\Phi-\kappa_{n} \tilde{z} S-\kappa_{n} \beta c t\right), \\
I_{3}^{\mathrm{CTR}}= & -\cos \left(\kappa_{n} \zeta\right)+C_{n 3} \cos \left[\Phi+\kappa_{n}(\zeta-d)\right],
\end{aligned}
$$


where

$$
\begin{aligned}
\left\{\begin{array}{l}
C_{n 1} \\
C_{n 21} \\
C_{n 22}
\end{array}\right\} & =\left\{\begin{array}{l}
\sin \left(\kappa_{n} d S\right)\left(1-\varepsilon^{2} S^{2}\right) \\
\varepsilon S-1 \\
\varepsilon S+1
\end{array}\right\} \frac{C_{n 3}}{2 \varepsilon S}, \\
C_{n 3} & =\left[1+\left(\frac{\varepsilon^{2} S^{2}-1}{2 \varepsilon S}\right)^{2} \sin ^{2}\left(\kappa_{n} d S\right)\right]^{-1 / 2}, \\
\Phi & =\operatorname{atan}\left[\frac{\varepsilon^{2} S^{2}+1}{2 \varepsilon S} \tan \left(\kappa_{n} d S\right)\right] .
\end{aligned}
$$

Consider the radiation in the area after the vacuum cavity (medium 3). Note that the first summand in $I_{3}^{\mathrm{CTR}}$ compensates for the Cherenkov radiation (12) in the domain $d<z<Z_{3}(t)$. Therefore, the total field can be approximately written in the form

$$
\begin{aligned}
E_{z 3} \approx & E_{z 3}^{\mathrm{CR}}+E_{z 3}^{\mathrm{CTR}} \\
= & \frac{-4 q}{a^{2} \varepsilon} \sum_{n=1}^{\infty} \frac{J_{0}\left(\frac{\chi_{n} r}{a}\right)}{J_{1}^{2}\left(\chi_{n}\right)} \tilde{\eta}\left(\omega_{0 n}^{(1)}\right) \\
& \times\left\{C_{n 3} \cos \left[\kappa_{n}(\zeta-d)+\Phi\right] \theta\left(Z_{3}-z\right)\right. \\
& \left.+\cos \left(\kappa_{n} \zeta\right) \theta\left(z-Z_{3}\right)\right\} .
\end{aligned}
$$

As a result, the amplitude of the field in the area $d<z<$ $Z_{3}(t)$ is determined by the coefficient $C_{n 3}$, and it depends periodically on the cavity lengths with maximums $C_{n 3 \max }=1$. These maximums occur at

$d=d_{n k}=\frac{\pi k}{\kappa_{n} S}=\frac{a \pi k \sqrt{\beta^{2} \varepsilon-1}}{\chi_{n} \sqrt{1-\beta^{2}(\varepsilon-1)}}, \quad k=1,2, \ldots$

For this length of the gap, the wave field is equal to the wakefield (12). Note that $C_{n 1}=0$ at (17); that is, the cavity does not reflect Cherenkov radiation. In the cavity, a standing wave arises at (17) and a resonant phenomenon takes place.

For ultrarelativistic bunches $(\gamma \gg 1)$, the above condition (17) is written in the form $d=a \pi k \sqrt{\varepsilon-1}\left(\chi_{n} \sqrt{2-\varepsilon}\right)^{-1}$. Naturally, this condition can be fulfilled for $\varepsilon<2$ only. (Note that if $\varepsilon>2$, then the $\mathrm{CR}$ of the ultrarelativistic bunch does not penetrate practically into the gap because of the total reflection from the first boundary).

It should be noticed that there is a special case when $C_{n 3}=1$ for any cavity length $d$. One can see from (14) that this holds true when $\varepsilon^{2} S^{2}=1$, which results in the relation

$$
\varepsilon=\varepsilon_{0}=\left(1+\sqrt{1+4 \beta^{2}}\right) / 2 \beta^{2} .
$$

To explain this phenomenon, consider wave of the CTR in the cavity determined by (7). This wave is proportional to

$$
J_{0}\left(\chi_{n} r / a\right) \exp \left[ \pm i \kappa_{n}(S \tilde{z} \mp \beta c t)\right]
$$

As consistent with Brillouin's concept, we deal with waves such as $\sim \exp \left[ \pm i \chi_{n} r / a \pm i \kappa_{n} S \tilde{z}-i \kappa_{n} \beta c t\right]$. One can see that the angle of incidence of these waves to the vacuumdielectric boundary is determined by the relation

$$
\left|\tan \theta_{i}\right|=\frac{\chi_{n}}{a \kappa_{n} S}=\frac{\sqrt{\beta^{2} \varepsilon-1}}{\sqrt{1-\beta^{2}(\varepsilon-1)}} .
$$

It can be shown that for $\varepsilon=\varepsilon_{0}$ this angle equals the Brewster angle $\left|\theta_{i}\right|=\theta_{B}=\operatorname{atan}\left(\sqrt{\varepsilon_{0}}\right)$. It is well known that a wave having the polarization under consideration does not reflect on the boundary at this incident angle.

Thus, for $\varepsilon=\varepsilon_{0}$ the CTR perfectly penetrates the boundary with no reflection for any gap length $d$. For ultrarelativistic bunches $(\gamma \gg 1)$, the optimal value of the permittivity equals $\varepsilon_{0}=(1+\sqrt{5}) / 2 \approx 1.62$. It is interesting that we can achieve coincidence of the CTR with the CR not only in amplitude but also in phase. Really, if we choose the length $d$ so that $\kappa_{n} d=\Phi+2 \pi l$ [ $\Phi$ is described by (15)], then both of the summands in (16) have the same phase, and furthermore, (16) is equivalent to (12).

So, we have found two cases [(17) and (18)] when all of the radiation is directed forward because the amplitude of the total field in the area after the cavity equals the wakefield amplitude $\left(C_{n 3}=1\right)$. In these situations the amplitudes of the reflected waves in the dielectric before the vacuum cavity are negligible $\left(C_{n 1}=0\right)$ [as can be seen from (14), (17), and (18)].

The behavior of the first modes of the normalized CTR amplitudes in media 1 and 3 for different lengths of the cavity $d / a$ and for different permittivities $\varepsilon$ are shown in Fig. 3 for some parameters of the problem, including the optimal ones. As can be seen, the CTR amplitude can be equal to the wakefield amplitude after a gap with a wide range of parameters (which was discussed above). Note that, here and further, we give computations for the first mode as an example only. The behaviors of the CTR amplitudes of other modes are analogous.

Another situation takes place in the case $\beta>\beta_{\mathrm{CT}}$, where the CTR effect is absent in the vacuum gap because of the total internal reflection of the Cherenkov waves from the first boundary. The contributions from the poles $\pm \omega_{0 n}^{(1)}$ to the free field give expressions that exist in the areas $|z|<z_{1}(t)$ and $z<z_{3}(t)$, where $z_{1}(t)=t \mathrm{v}_{\mathrm{g} 1}, \quad t>0$; $z_{3}(t)=d+[t-d /(\beta c)] \mathrm{v}_{\mathrm{g} 1}, t>d /(\beta c), \mathrm{v}_{\mathrm{g} 1}$ is described by (9). One can obtain that the $n$-th mode of the normalized amplitude (14) in medium 3 decreases with an increase in $d$ and in $\varepsilon>2$ for ultrarelativistic bunches:

$$
C_{n 3}=1-\frac{(\varepsilon-1)}{8 \varepsilon^{2}}\left(1+\varepsilon-\varepsilon^{2}\right)^{2} \chi_{n}^{2}\left(\frac{d}{a}\right)^{2}+O\left[\left(\frac{d}{a}\right)^{3}\right] .
$$




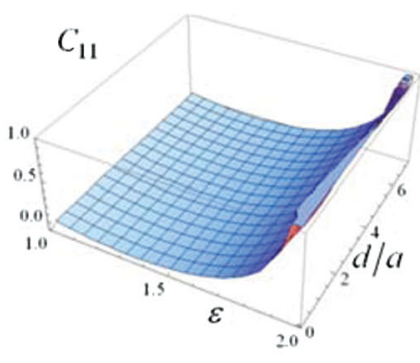

(a)

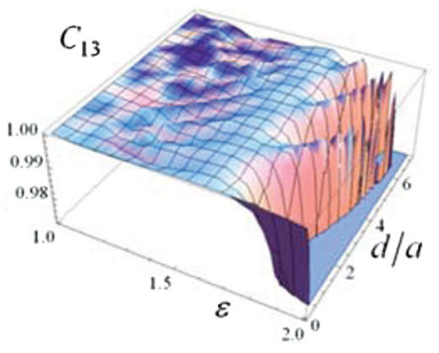

(d)

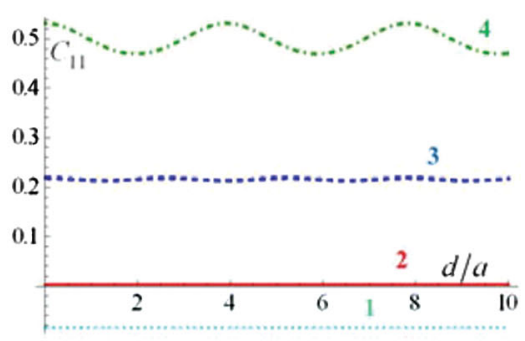

(b)

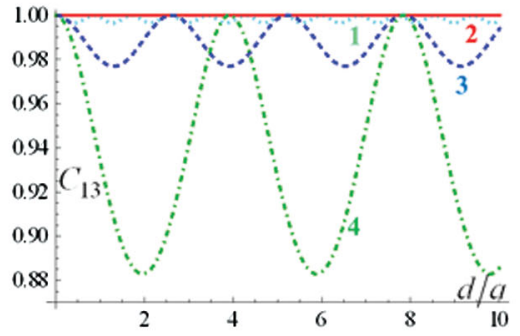

(e)

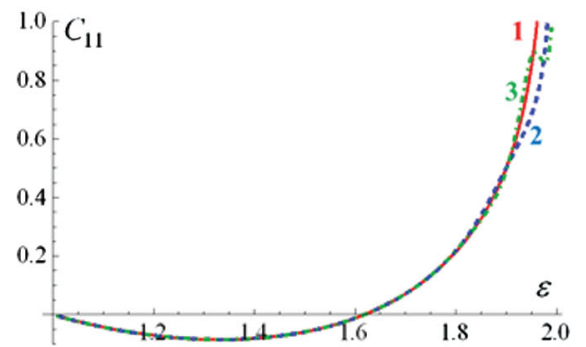

(c)

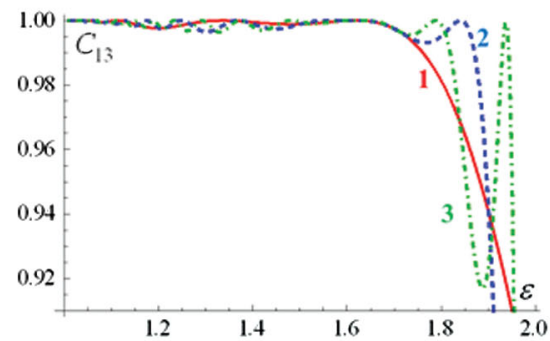

(f)

FIG. 3. The dependences of the first modes of the normalized CTR amplitudes in media 1 ( $C_{11}$, top) and 3 ( $C_{13}$, bottom) on the cavity length $d$ and on the permittivity $\varepsilon$; parts (b) and (e): cyan dotted line 1 is for $\varepsilon=1.3$, red solid line 2 is for $\varepsilon=1.62$, blue dashed line 3 is for $\varepsilon=1.8$ and green dot-dashed line 4 is for $\varepsilon=1.9$; parts (c) and (f): red solid line 1 is for $d / a=0.93$, blue dashed line 2 is for $d / a=3$ and green dot-dashed line 3 is for $d / a=5.035$; it is assumed that $\beta \approx 1$.

However, a major contribution to the free field makes the TR, which can be evaluated for small gaps with the steepest descent technique [11]. It can be seen that the TR is absent in a zero-order approximation $(d / a)^{0}$, and the main part of the TR for small cavities is proportional to $(d / a)^{1}$. The TR exists only in the areas $|z|<z_{01}(t)$ and $z<z_{03}(t)$, where $z_{01}(t)=t \mathrm{v}_{\mathrm{ph} 1}, t>0 ; z_{03}(t)=d+(t-d / c) \mathrm{v}_{\mathrm{ph} 1}, t>d / c$, and

$$
\mathrm{v}_{\mathrm{ph} 1}=c / \sqrt{\varepsilon}
$$

is the phase velocity of the TR in a dielectric. For the TR part of the free field (3), one can derive the following expression:

$$
\begin{aligned}
I_{z 1,3}^{\mathrm{TR}} \approx & d(\varepsilon-1) \sqrt{2 \pi \varepsilon c R_{1,3} \omega_{n}^{-1}} \\
& \times \operatorname{Re}\left\{\tilde{f}_{1,3}\left(\omega_{01,3}\right) \exp \left[-i\left(\frac{\omega_{n} R_{1,3}}{c}+\frac{\pi}{4}\right)\right]\right\},
\end{aligned}
$$

where $\quad \omega_{n} R_{1,3} c^{-1} \gg 1, \quad \omega_{01,3}=\omega_{n} c t\left(\varepsilon R_{1,3}\right)^{-1}, \quad R_{1}=$ $\sqrt{c^{2} t^{2} \varepsilon^{-1}-z^{2}}$, and $R_{3}=\sqrt{c^{2} t^{2} \varepsilon^{-1}-\tilde{z}^{2}}$. Therefore, the magnitudes of the TR in media 1 and 3 increase with increases in $d$.

\section{NUMERICAL RESULTS AND DISCUSSION}

The numerical calculations of the free field (3) are based on an algorithm that uses a certain separation between the integration path and the singularities of the integrands.
We take into account a small absorption in the media, that is, we assume that the permittivity is complex: $\varepsilon_{j}=\varepsilon_{j}+i \varepsilon_{d}, j=1,2,3$. It can be seen that this assumption results in the displacement of the singularities into the lower half plane of $(\omega)$, as shown in Fig. 1. As a consequence, the integrands of (3) have relatively smooth behaviors, and one can perform the computations with very good accuracy by optimizing the parameters of the integrations. All of the results presented in Figs. 4-6 are for ultrarelativistic Gaussian bunches with $\gamma=7$ in the two different situations $\varepsilon<2$ and $\varepsilon>2$, which correspond to different physical phenomena. Note that we show here the first mode because this mode plays usually the main role in application; the behavior of other modes is similar.

In Fig. 4, the longitudinal component of the first mode of the total field is shown for different time moments, the dielectric permittivity $\varepsilon<2$, and for the one of the optimal cavity length. The CTR found analytically (7) is presented as well. One can see a very good coincidence between the analytical and exact solutions in all of the media (the dielectrics and the vacuum gap). The TR on the cavity is negligibly small in almost all regions, and the CTR together with the CR are the main parts of the total field.

As the condition for the CTR effect is fulfilled $\left(\beta_{C}<\beta<\beta_{\mathrm{CT}}\right)$, the $\mathrm{CR}$ penetrates into the vacuum cavity and through the second boundary, which results in restoration of the wakefield in the dielectric after the gap [Figs. 4(a), 4(b), 4(d), and 4(e)] for the optimal parameters. It should be recalled that in the case of a single vacuumdielectric boundary, there is compensation for the wakefield 


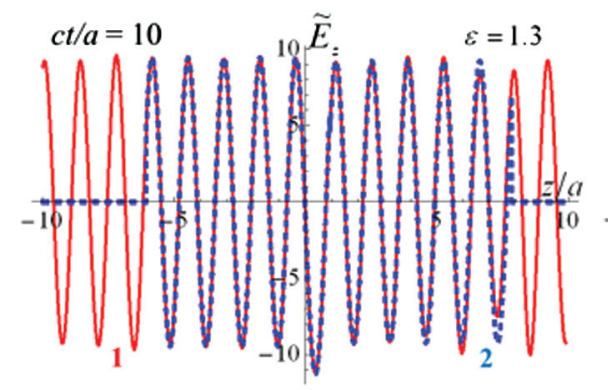

(a)

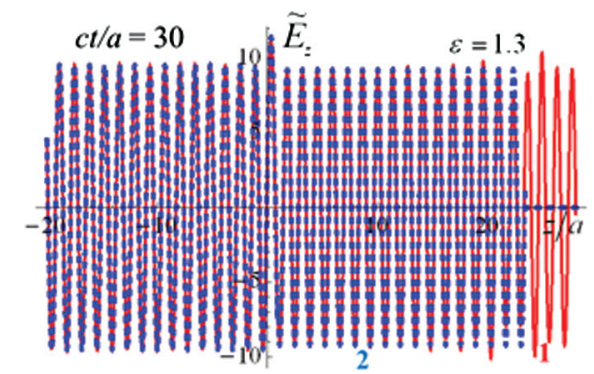

(d)

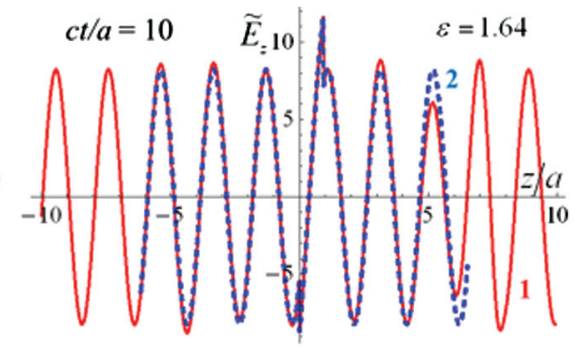

(b)

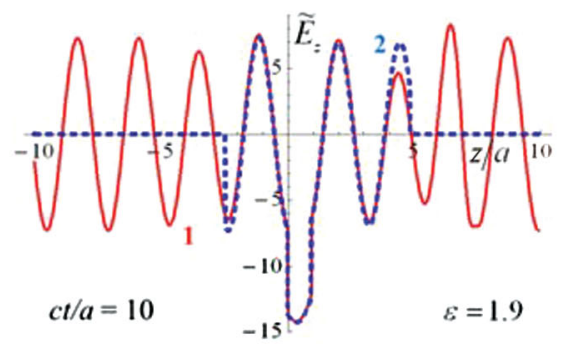

(c)

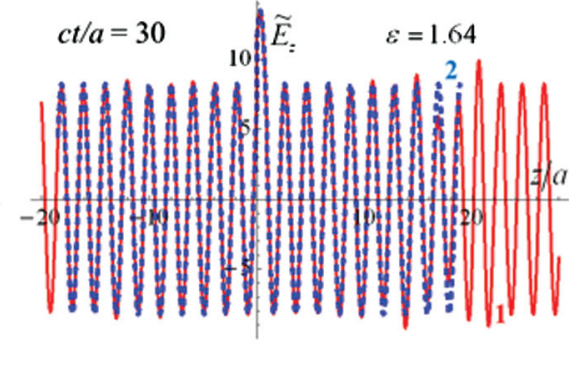

(e)

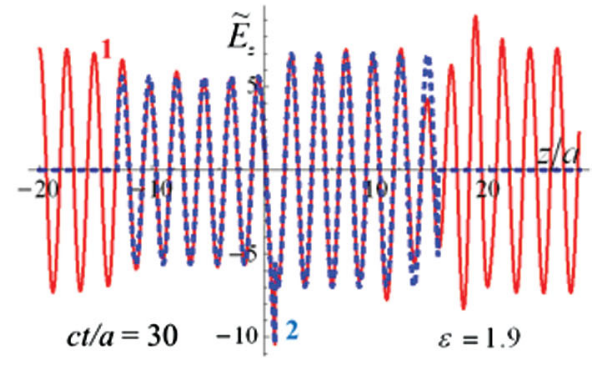

(f)

FIG. 4. The dependence of normalized longitudinal component $\tilde{E}_{z}=E_{z} a^{2} / q$ of the first mode of the total field (continuous line 1) and the CTR together with the forced field (dashed line 2) on distance $z / a$ for different dimensionless times $c t / a$ and permittivities $\varepsilon$; $d / a=0.93, \sigma / a=0.1, \omega_{0}=2 \pi \times 10 \mathrm{GHz}, \varepsilon_{d}=10^{-4}, \gamma=7$, and $a=5 \mathrm{~mm}$. The sighting point is on the waveguide axis $r=0$.

in some area after the border [7]. If the optimality is broken [Figs. 4(c) and 4(f)], then there is significant backwards radiation, and the restoration of the wakefield does not occur in full. The boundary of the area of this phenomenon moves with the group velocity of the CTR in the dielectric area (9).

If $\varepsilon>2$ and the CTR effect is absent in the vacuum gap, then there is no restoration of the wakefield for large

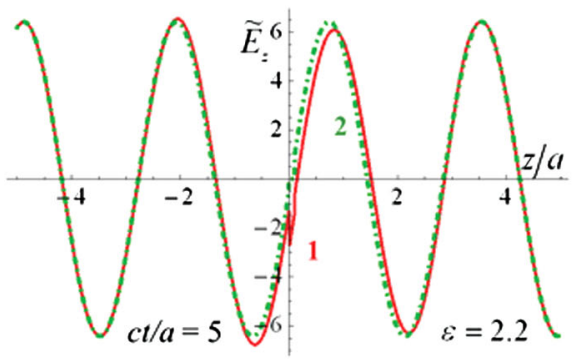

(a)

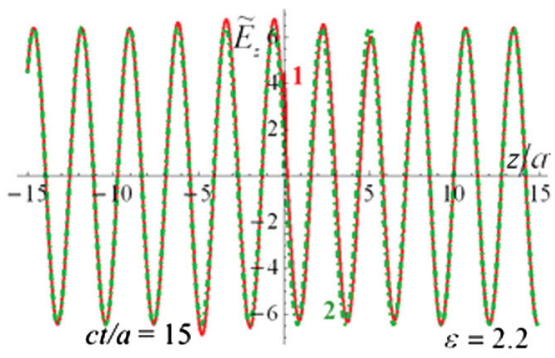

(d)

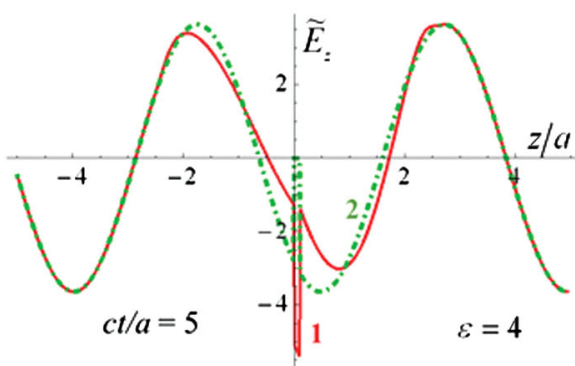

(b)

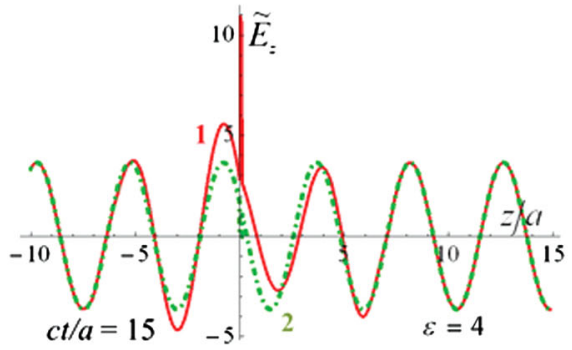

(e)

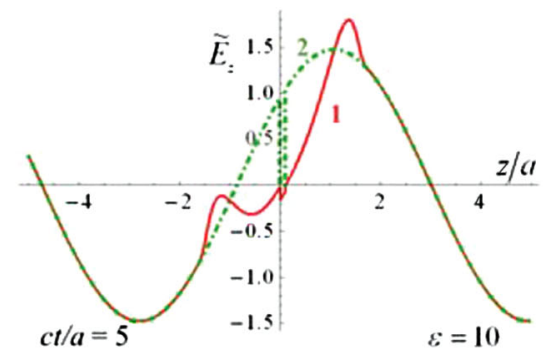

(c)

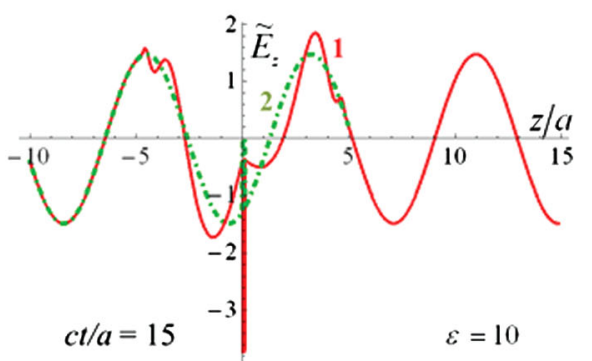

(f)

FIG. 5. The dependence of the normalized longitudinal component $\tilde{E}_{z}=E_{z} a^{2} / q$ of the first mode of the total field (continuous red line 1) and the wakefield (dot-dashed green line 2) on distance $z / a$ for different dimensionless times $c t / a$ and permittivities $\varepsilon$; $d / a=0.1, \sigma / a=0.1, \omega_{0}=2 \pi \times 10 \mathrm{GHz}, \varepsilon_{d}=10^{-4}, \gamma=7$, and $a=5 \mathrm{~mm}$. The sighting point is on the waveguide axis $r=0$. 


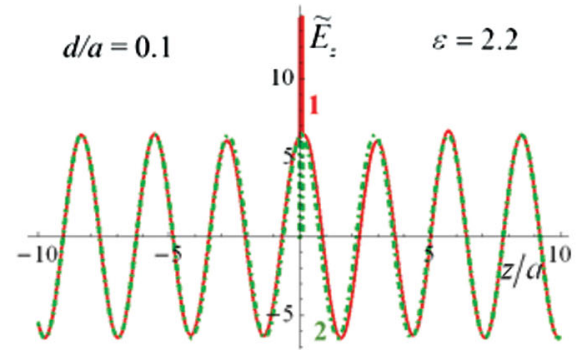

(a)

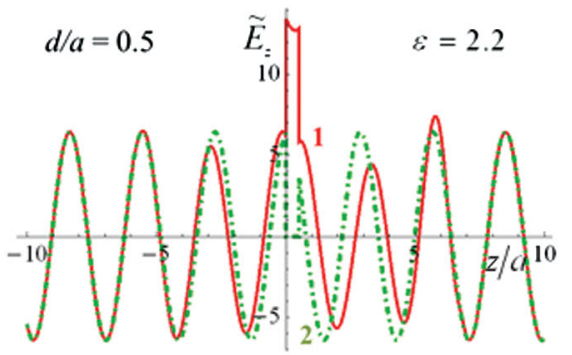

(d)

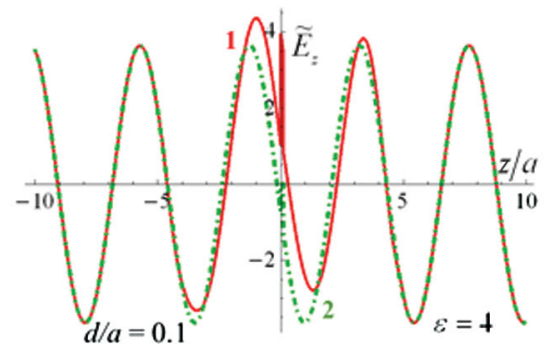

(b)

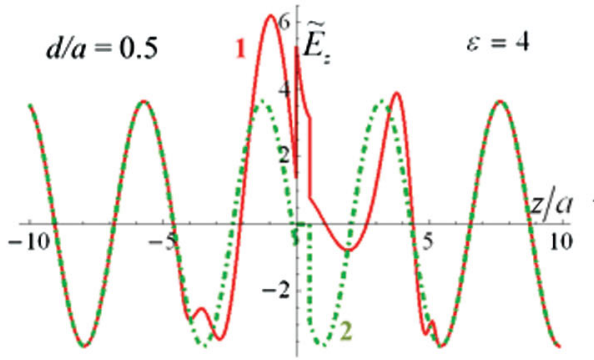

(e)

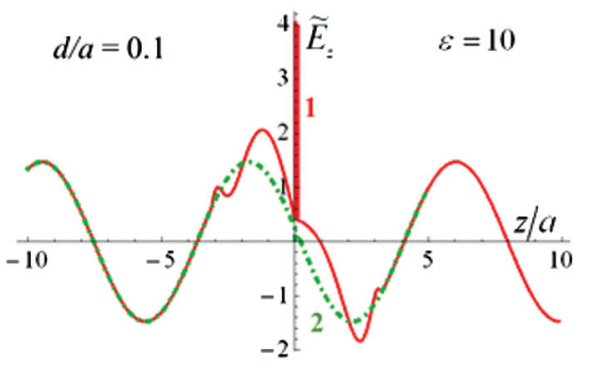

(c)

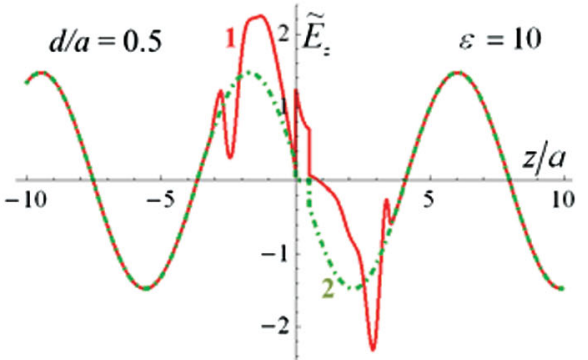

(f)

FIG. 6. The same as in Fig. 4 for different lengths of the gap $d / a$ and permittivities $\varepsilon ; c t / a=10$.

lengths of the gap. In this situation, the TR on the cavity becomes significant, and the investigation of the influence of the vacuum gap on the wakefield is important. In Figs. 5 and 6, the longitudinal component of the first mode of the total field and of the wakefield are presented for different time moments, different dielectric permittivities $\varepsilon$ and different cavity lengths $d$. One can see that relatively small gaps are minimally influential on the wakefield for dielectrics with relatively small permittivity [Figs. 5(a), 5(d) and 6(a)]. The influence of the cavity increases with increases in $\varepsilon$ [Figs. 5(b), 5(c), 5(e), 5(f), 6(b), and 6(c)] and in $d$ [Figs. 6(d), 6(e), and 6(f)]. Furthermore, the domain of this influence increases with increases in the time moments [Figs. 5(c), 5(f), and 6(c)] and is defined by the phase velocity of the TR in dielectric (20).

\section{CONCLUSION}

In summary, we have investigated the electromagnetic field of a bunch moving in a dielectric loaded waveguide and crossing a vacuum cavity. This study includes results derived both analytically and numerically and reveals two different situations. One of these situations is characterized by the CTR existing in the vacuum gap, but this phenomenon is absent in the other situation. For ultrarelativistic bunches, these situations correspond to the permittivities of the dielectric $\varepsilon<2$ and $\varepsilon>2$ accordingly.

If the CTR exists in the vacuum cavity, then it influences the field in the third (dielectric) segment of the waveguide. In this case, one can choose certain optimal parameters that enable to restore the field amplitude in the whole area after the vacuum gap. These parameters can be connected with the resonance effect as well as with the Brewster phenomenon. The TR is negligibly small in this situation, and the CTR and CR are the main parts of the total field. The border of area of the CTR moves with the group velocity in the dielectric area. As a result, the CTR and the CR are joined in the area after the gap, and furthermore, one can obtain the radiation with the same amplitude as in the infinite homogeneous waveguide loaded with the dielectric. In certain situations, it is possible to achieve coincidences in the phases of the CTR and CR as well.

If the CTR effect is absent in the vacuum cavity, then the TR is significant. The influence of the vacuum cavity increases with increases in $\varepsilon$ and $d$. The domains of this influence are determined by the phase velocity of the TR in the dielectric.

\section{ACKNOWLEDGMENTS}

This research was supported by Saint Petersburg State University (Grant No. 11.0.61.2010).

[1] W. Gai, AIP Conf. Proc. 1086, 3 (2009).

[2] A. M. Cook, R. Tikhoplav, S. Y. Tochitsky, G. Travish, O. B. Williams, and J. B. Rosenzweig, Phys. Rev. Lett. 103, 095003 (2009).

[3] A. Kanareykin, J. Phys. Conf. Ser. 236, 012032 (2010).

[4] K. A. Barsukov, Zh. Tekh. Fiz. 30, 1337 (1960).

[5] K. A. Barsukov, E. D. Gazazian, and E. M. Laziev, Izv. Vysshykh. Uchebn. Zaved., Radiofiz. 15, 191 (1972).

[6] T. Yu. Alekhina and A. V. Tyukhtin, J. Phys. Conf. Ser. 517, 012012 (2014). 
[7] T. Yu. Alekhina and A. V. Tyukhtin, J. Phys. Conf. Ser. 357, 012010 (2012).

[8] T. Yu. Alekhina and A. V. Tyukhtin, Phys. Rev. ST Accel. Beams 15, 091302 (2012).

[9] V. L. Ginzburg and V. N. Tsytovich, Transition Radiation and Transition Scattering (Hilger, London, 1990).
[10] B. M. Bolotovskii, Sov. Phys. Usp. 4, 781 (1962).

[11] B. Felsen and N. Marcuvitz, Radiation and Scattering of Waves (Wiley Interscience, New Jersey, 2003).

[12] T. Yu. Alekhina and A. V. Tyukhtin, Phys. Rev. E 83, 066401 (2011). 